\title{
Sensitive Observations of Radio Recombination Lines in Orion and W51: The Data and Detection of Systematic Recombination Line Blueshifts Proportional to Impact Broadening
}

\author{
M.B. Bell ${ }^{1}$, L.W. Avery², J.M. MacLeod ${ }^{2}$, J.P. Vallée ${ }^{2}$
}

\begin{abstract}
Sensitive spectral observations made in two frequency bands near 6.0 and $17.6 \mathrm{GHz}$ are described for Orion and W51. Using frequency switching we were able to achieve a dynamic range in excess of 10,000 without fitting sinusoidal or polynomial baselines. This enabled us to detect lines as weak as $\mathrm{T}_{A} \sim 1 \mathrm{mK}$ in these strong continuum sources. Hydrogen recombination lines with $\Delta n$ as high as 25 have been detected in Orion. In the Orion data, where the lines are stronger, we have also detected a systematic shift in the line center frequencies proportional to linewidth that cannot be explained by normal optical depth effects.
\end{abstract}

Subject headings: HII regions - ISM: abundances — radio lines: ISM

\section{Introduction}

In a recent paper Gordon (2008) has discussed how radio recombination lines can be useful tools for astronomers and physicists. Because of their high spectral density, observations in a narrow frequency band typically encompass lines corresponding to a wide range of $n$ values, where $n$ is the principal quantum number. Provided the sensitivity is high enough, such observations allow the study of impact broadening and source analysis based upon many transitions, free of systematic errors due to differences in beam sizes, calibration errors and telescope efficiencies. Another advantage of observing lines of different $n$ in the same window is that the optical depth of the radio continuum is the same for all lines (Churchwell 1971: Lockman and Brown 1975; Peimbert et al. 1992). With the same absorption effects operating in each line it is possible to make a more reliable investigation of line broadening mechanisms such as turbulence and electron impact broadening Griem

\footnotetext{
${ }^{1}$ Herzberg Institute of Astrophysics, National Research Council of Canada, 100 Sussex Drive, Ottawa, ON, Canada K1A 0R6; morley.bell@nrc-cnrc.gc.ca

${ }^{2}$ Herzberg Institute of Astrophysics, National Research Council of Canada, 5071 West Saanich Road, Victoria, BC V9E 2E7
}

1967).

We report here the results obtained by making small-offset, frequency-switched observations (hereafter referred to as SOFS observations) at a fixed frequency, with a relatively wide, instantaneous bandwidths $(140 \mathrm{MHz}$ at 17.6 $\mathrm{GHz}$ and $70 \mathrm{MHz}$ at $6.0 \mathrm{GHz}$ ), in W51 and Orion A, two sources with strong continuua. The source coordinates used were R.A.(1950) = $05^{h} 32^{m} 50^{s} ; \operatorname{Dec}(1950)=-05^{\circ} 25^{\prime} 12^{\prime \prime}$ for Orion $\mathrm{A}$ and R.A.(1950) $=19^{h} 21^{m} 26^{s} ; \operatorname{Dec}(1950)=$ $14^{\circ} 24^{\prime} 40^{\prime \prime}$ for W51(Main). Our observations were carried out using the NRAO ${ }^{1} 140$-foot $(43 \mathrm{~m}$ ) telescope. The data at $6.0 \mathrm{GHz}$ were obtained in 1990 August (W51) and 1992 April (Orion); the data at $17.6 \mathrm{GHz}$ in 1991 January.

We present these data for publication at this time for two main reasons. First, in the ten years since we first described our observing and data reduction techniques (Bell 1997), and reported a mysterious reduction in impact broadening above $n=200$ (Bell et al. 2000), we have had many requests from astronomers asking to see the data. Second, we fully anticipated that

\footnotetext{
${ }^{3}$ The National Radio Astronomy Observatory (NRAO) is operated by Associated Universities Inc., under agreement with the National Science Foundation.
} 
when the Green Bank Telescope (GBT) was commissioned ten years ago, it would soon be used either to confirm or to rule out our earlier findings. This has not happened. To our knowledge, even the GBT, with its offset feed designed to reduce the feed-to-primary dish reflection problem, and its extensive receiver-room shielding to control local interference problems, has not obtained position-switched results over wide windows close to the $10,000 / 1$ dynamic range we achieved with the 140-foot telescope using frequency switching. We hope by presenting our data it might encourage someone to use the GBT to try to explain the mysterious line narrowing problem above quantum levels of $n=200$ that we previously reported.

Here, the spectra obtained for each source are presented at both observing frequencies. The peak antenna temperatures and linewidths (FWHM) are tabulated. For sources with a strong continuum, frequency switching offers the opportunity to observe baseline-free spectral windows that are many times wider than the continuous window widths obtainable using position switching. Although the same correlator window widths can be used with both techniques, because of the need to fit high-order polynomials to position-switched data, only small portions of the total observing window can be processed at a time.

Observations have been reported previously of recombination lines arising from transitions between levels separated by $\Delta n$ up to at least 14 (Balser et al. 1994, Rood et al. 1984; Bania et al. 1987: Bell 1995: Davies 1971:|Smirnov et al. 1984). Here we report recombination lines with $\Delta n$ values possibly as high as $\Delta n=25$. Throughout this paper the principal quantum number $n$ represents the lower level of a given transition whose quantum designation is given as $(n, \Delta n)$.

In $\S 2$ we describe the receivers and observing technique. In $\S 3.1$ we describe the spectra obtained for both sources at $17.6 \mathrm{GHz}$, including molecular lines. In $\S 3.2$ the spectra obtained at $6 \mathrm{GHz}$ are described. In $\S 3.3$ some of the main molecular lines in our observing window are discussed. In $\S 4$ we describe the RRLs listed in Tables $2,3,4,6$, and other, non-recombination lines listed in Table 7, as well as how the corrected values have been obtained. In $\S 5$ the center frequencies of the RRLs in Orion at $6 \mathrm{GHz}$ are examined and shown to exhibit a systematic blueshift as $n$ and $\Delta n$ in- crease. A brief description of the correction curves used to recover the true line parameters is included in Appendix A.

\section{Observations}

As reported by Bell and Feldman (1991), it turned out to be necessary to make off-source integrations of equal duration, at least for the first several hours to ensure that the spectra were not contaminated by telluric lines or interference. Using frequency-switching, these lines, that otherwise disappear with off-source subtraction, are readily detected. However, this possible spectral contamination is not too serious since telluric lines can usually be identified, and even time-variable weak interference can be discovered by dividing the data into two halves and subtracting them. All results were obtained using relatively short, 4minute $\mathrm{ON}-\mathrm{OFF}$ integration periods. To reduce the fundamental telescope ripple we also used $\lambda / 8$ defocussing.

The autocorrelator was configured to give two series banks of $80 \mathrm{MHz}$ and 256 channels at 17.6 $\mathrm{GHz}$ which gave channel separations of 0.3125 $\mathrm{MHz}$ or $5.32 \mathrm{~km} \mathrm{~s}^{-1}$. The bank center frequencies were separated by $70 \mathrm{MHz}$, allowing a frequency overlap of $10 \mathrm{MHz}$ to accommodate autocorrelator edge effects. The observing window thus covered $140 \mathrm{MHz}$, and with cold-sky system temperatures between $50-60 \mathrm{~K}$ at $17.6 \mathrm{GHz}$ the desired sensitivity was obtained in six transits. Telescope pointing was updated every 90 minutes using standard pointing sources. The relevant telescope beamwidths and efficiencies are given in Table 1.

At $6.0 \mathrm{GHz}$ the autocorrelator was configured to give two series banks of $40 \mathrm{MHz}$ width and 512 channels which gave channel separations of $0.0781 \mathrm{MHz}$ or $3.9 \mathrm{~km} \mathrm{~s}^{-1}$. The frequency offsets used at 17.6 and $6.0 \mathrm{GHz}$ were $\pm 0.9375 \mathrm{MHz}$ and 0.3125 respectively, (which correspond.to frequency-switched intervals of 32 and $31.2 \mathrm{~km}$ $\mathrm{s}^{-1}$ ). Although a slightly larger offset would have been desirable, the baseline degradation with increasing offset ruled this out. The flatness of the baseline and hence the line-detecting ability of the observing technique was felt to be more important than the loss of line parameter information which may have resulted, especially since 
the latter can be recovered. Also, removing the frequency-switched reference lines so that Gaussians can be fitted requires that the baselines be flat.

The LINECLEAN program used in the reduction process employed a scaled version of the signal line to remove the reference lines. It is described in detail in Bell (1997). In the reduction process the known frequencies of all recombination lines, up to $\Delta n=20$ at $17.6 \mathrm{GHz}$ and up to $\Delta n=25$ at $6 \mathrm{GHz}$, were examined and were cleaned if they showed evidence of a line. Very weak features that were confused with other features were not cleaned. A complete discussion of the procedures used to obtain flat baselines with the SOFS observing technique, without having to fit polynomial or sinusoidal baselines, is given in Bell (1997).

\section{Results}

\subsection{The $17.6 \mathrm{GHz}$ data}

The spectrum of Orion A near $17.6 \mathrm{GHz}$ is shown in Figure 1 on a scale that reveals the weakest lines. No sinewaves or polynomials have been removed from this spectrum even though the continuum brightness temperature of the source is in excess of $40 \mathrm{~K}$. A list of the detected recombination lines is presented in Table 2. It is immediately apparent in Figure 1 that the baseline structure, even at the $1 \mathrm{mK}$ level, is limited mainly by thermal noise. The only obvious nonrecombination line, detected in this window, that is of reasonable width, is at $17595.3 \mathrm{MHz}$. This corresponds to the overlapping $38_{16,23}-37_{17,20}$ and $38_{16,22}-37_{17,21}$ transitions of $\mathrm{CH}_{3} \mathrm{COOH}$ which is a common molecule in Orion. The $2 \mathrm{p} \sigma_{\mu}(\mathrm{V}=0, \mathrm{~N}=2, \mathrm{G}=\mathrm{l} / 2)-\mathrm{ls} \sigma_{g}(\mathrm{~V}=19, \mathrm{~N}=\mathrm{l}, \mathrm{G}=\mathrm{l} / 2)$ and $2 \mathrm{p} \sigma_{\mu}(\mathrm{V}=0, \mathrm{~N}=2, \mathrm{G}=3 / 2)-\mathrm{l} \sigma_{g}(\mathrm{~V}=19, \mathrm{~N}=\mathrm{l}, \mathrm{G}=3 / 2)$ transitions of $\mathrm{H}_{2}^{+}$at $17604.3 \pm 0.5$ and $17610.3 \pm 0.5$ $\mathrm{GHz}$ respectively (Carrington et al. 1993), also fall inside this window and were one of the main reasons for its choice. These results provide a very sensitive upper limit to the amount of $\mathrm{H}_{2}^{+}$in Orion A. Although features at these frequencies might be confused with the $\mathrm{H}(163,13)$ line at $17606.8 \mathrm{MHz}$, any $\mathrm{H}_{2}^{+}$line present is unlikely to be stronger than $\mathrm{T}_{A} \sim 3 \mathrm{mK}$.

The 17.6 GHz spectrum of W51 is shown in Figure 2 and a list of the detected lines is given in
Table 3. Although the spectrum is similar in appearance to Orion $\mathrm{A}$, the recombination lines are roughly $50 \%$ weaker. As in the Orion A spectrum, there is no evidence for $\mathrm{H}_{2}^{+}$to a level of $\sim 1 \mathrm{mK}$.

\subsection{The $6 \mathrm{GHz}$ data}

The spectrum of Orion $\mathrm{A}$ at $6 \mathrm{GHz}$ is presented in Figure 3 with the vertical scale adjusted so that a line of strength $\mathrm{T}_{A}=1 \mathrm{mK}$ is visible. The sensitivity is significantly better in the high-frequency half of the spectrum due to a change in receiver noise across the $70 \mathrm{MHz}$ bandwidth. The locations of all recombination lines with $\Delta n<25$ are indicated whether or not a line has been detected. There is a strong, variable feature near $6005 \mathrm{MHz}$ that has prevented the detection of weak lines within at least $2 \mathrm{MHz}$ to either side.

Although it was assumed to be interference, it is worth noting that the ${ }^{2} \Pi_{3 / 2}\left(2.5_{1.5,1.0}-2.5_{0.5,0.0}\right)$ transition of ${ }^{17} \mathrm{OH}$ is located at $6005.6 \mathrm{MHz}$ and the possibility that this line may be masering cannot be ruled out. A list of the lines detected is given in Table 4 . The notes to Table 4 are given in Table 5. Although 41 lines are listed, only the rest frequency is given if the line is confused with a stronger one. About ten percent of the lines appear to be molecular in origin and some of these are discussed in more detail in $\S 3.3$ below and in Fig 6. The $\mathrm{H}(191,10)$ line near $8 \mathrm{GHz}$, was detected by Rood et al. (1984), with $\mathrm{T}_{A}=6 \mathrm{mK}$. For comparison purposes the $\Delta n=9$ line at $6 \mathrm{GHz}$ in Orion reported here in Table 4 , has corrected peak antenna temperature of $\mathrm{T}_{A}=12.7 \mathrm{mK}$ (see $\S 4$ for a brief description of how the corrected peak antenna temperatures are obtained). It is not immediately obvious why the $\mathrm{H}(213,14)$ line near 8664 $\mathrm{MHz}$ was not visible in the spectrum of Orion obtained by Bania et al. (1987), or why they did not detect the $\mathrm{H}(203,12)$ or $\mathrm{H}(208,13)$ lines, especially in M17S, but these failures might have resulted from the fact that their data required the removal of a 12th order polynomial from the baseline.

The spectrum of W51 obtained at $6 \mathrm{GHz}$ is presented in Figs 4 and 5, with the vertical scale adjusted to show the $1 \mathrm{mK}$ level. Again there is no baseline structure apparent above the level of the thermal noise, except within the cleaned region of the stronger lines and no baselines have been removed. Recombination lines up to at least $\Delta n=$ 14 appear to be visible when they are located in 
unconfused regions. A list of the detected lines is given in Table 6 . The corrected peak antenna temperature for the $\mathrm{H}(194,7)$ line is $11.5 \mathrm{mK}$ in Table 6 , which shows good agreement with the $\mathrm{T}_{A}=14.1$ and $16.0 \mathrm{mK}$ reported by Bania et al, (1987) for the $\mathrm{H}(171,7)$ and $\mathrm{H}(170,7)$ lines in this source, using the same telescope.

In Table 7 we have listed similar information for the Helium recombination lines detected in W51 in the $6 \mathrm{GHz}$ window. Table 8 lists the frequencies and antenna temperatures of all molecular lines detected in W51 at $6 \mathrm{GHz}$.

\subsection{Molecular Lines}

Three components of the ${ }^{2} \Pi_{3 / 2}$ transition of ${ }^{16} \mathrm{OH}, \quad\left(2.5_{1.5,-1,1}-2.5_{1.5,1,3}\right),\left(2.5_{1.5,-1,2}-\right.$ $\left.2.5_{1.5,1,2}\right)$, and $\left(2.5_{1.5,-1,3}-2.5_{1.5,1,3}\right)$, near 6016, 6030 , and $6035 \mathrm{MHz}$ respectively, are present in the spectrum in Fig. 3 and are found to be redshifted by $\sim 22 \mathrm{~km} \mathrm{~s}^{-1}$, which is the velocity of the strongest outflow component near IRc2 (see e.g. Hansen and Johnson (1983). The weakest falls near the $\mathrm{H}(217,10)$ line.

There are also 3 Q-Branch transitions of NO in this spectrum, $\left(18.5_{0.5,-1.0,19.5}-18.5_{0.5,1.0,19.5}\right)$, $\left(19.5_{0.5,1.0,18.5}-19.5_{0.5,-1.0,18.5}\right)$, and $\left(19.5_{0.5,1.0,18.5}\right.$ - $\left.19.5_{0.5,-1.0,19.5}\right)$. Although the last of these is coincident with the $\mathrm{OH}$ component near $6035 \mathrm{MHz}$, the remaining two fall in relatively unconfused regions near 5981 and $5993 \mathrm{MHz}$ (see Table 4) and both show weak emission, also redshifted by an amount similar to that found for $\mathrm{OH}$.

The three lines of $\mathrm{OH}$ are also present in Figure 5 and are considerably narrower than the recombination lines, and unresolved. The weakest $\mathrm{OH}$ line at $6016.7 \mathrm{MHz}$ is seen in absorption and falls on top of the $\mathrm{H}(217,10)$ line. The two stronger $\mathrm{OH}$ lines, near 6030 and $6035 \mathrm{MHz}$, show both emission and absorption with the absorption component occurring in the red wing of the main emission feature. This can be seen more clearly in Figure 6 which shows an expanded view of the spectrum in the vicinity of the two strongest $\mathrm{OH}$ lines. The $\mathrm{H}(184,6)$ line in this spectrum may also show evidence for structure in its red wing, although this needs to be confirmed.

\section{Corrected Line Parameters}

In Table 2 we have listed in columns 1-3 all the recombination lines in Orion A with $\Delta n<20$ that fall in the $17.6 \mathrm{GHz}$ window, along with the $\mathrm{H} \alpha$ and $\mathrm{H} \beta$ lines near 17.99 and $18.94 \mathrm{GHz}$ respectively. The peak strengths and linewidths (FWHM) were determined from Gaussian fits using DRAWSPEC and have been included along with 'corrected' values. The corrected values are obtained using the correction curves in Figures 12 and 13 (see also Appendix A). These adjustments are necessary to remove the effects introduced by frequency switching with small offsets. Similar numbers for W51 are given in Table 3. In Table 4 we list in column 1 the frequencies of all $6 \mathrm{GHz}$ lines detected in Orion along with the frequencies of all the recombination lines that fall inside the observing window (for $\Delta n<25$ ). If the line was detected, its observed frequency is listed in column 2. Line identifications are listed in column 3. The observed antenna temperature is listed in column 4 and the antenna temperature, corrected for frequency-switched effects, is listed in column 5. The linewidth (FWHM) is included in columns 6 and 7 (frequency and velocity respectively). The corrected linewidths are given in column 8. Notes in column 9 are explained in Table 5. Table 6 lists the Hydrogen recombination lines detected in W51 and Table 7 lists the Helium recombination lines in W51. Table 8 lists the other, non-recombination lines detected in W51.

\section{Line Center Frequencies}

Figures 7, 8, 9, and 10 show lines from all relatively unconfused regions of the $6 \mathrm{GHz}$ Orion spectrum that contain a recombination line $(\Delta n<$ $26)$. Each spectrum has the same incremental frequency scale and the rest frequency of each line is indicated by the vertical solid line. The vertical dashed line indicates the line center frequency obtained from Gaussian fitting. For lines with $n<200(\Delta n<10)$ there is a systematic shift of the line to higher frequency (blueshift) as $n$, (or $\Delta n$ ), increases. This shift is also present in previously published data and, in Fig. 11, the differences between the observed and rest frequencies reported here are compared to line frequencies reported by others (Smirnov et al. 1984; Rood et al. 1984) for $\Delta n<10$. Since all measurements were made near 
the same frequency, the shifts cannot be due to normal optical depth effects related to changes in observing wavelength.

\section{Linewidths vs $n$}

Griem 1967, 1974) has shown that significant line broadening due to electron collisions can be expected in high-level Rydberg-Rydberg transitions that originate in regions of relatively high electron density. The broadening is proportional to $n^{4}$. Examination of Tables 4 and 5, however, shows that our measured linewidths begin to decrease again for $n>200$. This discrepancy is discussed in more detail in Bell et al. (2000), who show that this effect also appears to be present in earlier data.

\section{Conclusions}

We have obtained sensitive spectral line observations of W51 and Orion in narrow (70 and $140 \mathrm{MHz}$ wide) frequency bands at 6.0 and 17.6 GHz. A total of 61 lines have been detected, of which about $10 \%$ are of molecular origin, $10 \%$ are unidentified and are assumed to have molecular carriers, and the remainder are recombination lines with $\Delta n$-values at least as high as 21 . We have used the frequency switching observing technique to make sensitive spectral observations of weak lines in sources with a strong continuum and wide linewidths, and conclude that this can be a powerful technique, especially for spectral line surveys of strong continuum sources and observations of extended regions. Finally, we have detected a shift in the line centers for hydrogen recombination lines that is proportional to impact broadening. The shift cannot be due to optical depth effects in an expanding nebula.

\section{Acknowledgments}

We thank the staff of the 140-foot telescope for their assistance in ensuring that things ran smoothly during the observing periods. We especially wish to thank George Seielstad, Green Bank site director when these observations were carried out, for seeing that adequate time was made available to complete this program. We also thank Pierre Brault for assistance with the data reduction and figure preparation. Finally, we thank an anonymous referee for several comments that helped to improve the organization of the paper. 


\section{REFERENCES}

Alexander, P., and Leahy, J.P. 1987, MNRAS, 225, 1

Balser, D. S., Bania, T.M., Brockway, C. J., Rood, R.T., and Wilson, T.L. 1994, ApJ, 430, 667

Bania, T. M., Rood, R. T., and Wilson, T. L. 1987, ApJ, 323, 30

Bell, M. B. 1995, ApJ, 453, 773

Bell, M. B. 1997, PASP, 109, 609

Bell, M.B., Avery, L.W., Seaquist, E.R. and Vallée, J.P. 2000, PASP, 112, 1236

Bell, M. B., and Feldman, P. A. 1991, ApJ, 367, L33

Carrington, A., Leach, C. A., Moss, R. K, Steimle, T. C., Viant, M. R., and West, Y.D. 1993, J. Chem. Soc. Faraday Trans., 89(4), 603

Churchwell, E. 1971, A\&A,15,90

Davies, R. D. 1971, ApJ, 163, 479

Griem, H. R. 1967, ApJ, 148, 547

Griem, H. R. 1974, Spectral Line Broadening by Plasmas, publ. (Academic Press, New York)

Gordon, M.A. 2008, Spectral Line Shapes, 19th International Conf. on Spectral Lines, Valladolid, Spain; AIP Conference Proceedings, Vol. 1058, p 273

Hansen, S.S., and Johnston, K.J. 1983, ApJ, 267, 625

Lockman, F.J. and Brown, R. L. 1975, ApJ, 201, 134

Peimbert, M., Rodriguez, L.F., Bania, T. M., Rood, R.T. and Wilson, T.L. 1992, ApJ, 395, 484

Rood, R.T., Bania, T.M., and Wilson, T.L. 1984, ApJ, 280, 629

Smirnov, G.T., Sorochenko, R.L, and Pankonin, V. 1984, A\&A, 135, 116

This 2-column preprint was prepared with the AAS LATEX macros v5.2. 
TABLE 1

Telescope, Receiver and Correlator Parameters.

\begin{tabular}{lll}
\hline \multicolumn{1}{c}{ Parameter } & $17.6 \mathrm{GHz}$ & $6.0 \mathrm{GHz}$ \\
\hline beamwidth (arcmin) & 1.7 & 5.17 \\
$\eta_{A}$ & $0.32 \pm 0.03$ & 0.52 \\
$\eta_{B}$ & $0.42 \pm 0.04$ & 0.65 \\
$\mathrm{~T}_{\text {sys }}$ & $50-60 \mathrm{~K}$ & $40-50 \mathrm{~K}$ \\
Channel width $(\mathrm{MHz})$ & 0.3125 & 0.07813 \\
Freq. sw. offset $(\mathrm{MHz})$ & 0.9375 & 0.3125 \\
W51 $\mathrm{V}_{L S R}$ assumed $\left(\mathrm{km} \mathrm{s}^{-1}\right)$ & 60 & 60 \\
Orion $\mathrm{V}_{L S R}$ assumed $\left(\mathrm{km} \mathrm{s}^{-1}\right)$ & 0 & 0 \\
\hline
\end{tabular}

TABLE 2

Hydrogen Recombination Lines in Orion A ${ }^{1}$ AT $17.6 \mathrm{GHz}(\Delta n<20)^{2}$.

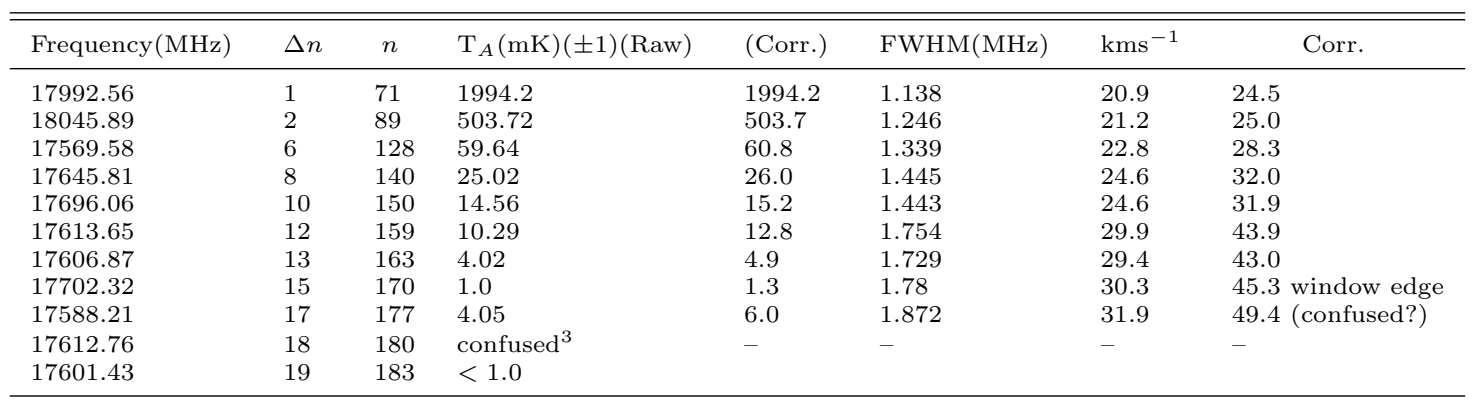

${ }^{1}$ assumes $\Delta \mathrm{V}($ Doppler $)=24.4 \mathrm{~km} \mathrm{~s}^{-1}$

${ }^{2}$ offset width $=32 \mathrm{~km} \mathrm{~s}^{-1}$

${ }^{3}$ confused with $\Delta n=12$ line

TABLE 3

Hydrogen Recombination Lines in W51 ${ }^{1}$ AT $17.6 \mathrm{GHz}(\Delta n<20)^{2}$.

\begin{tabular}{llllllll}
\hline \hline Frequency $(\mathrm{MHz})$ & $\Delta n$ & $n$ & $\mathrm{~T}_{A}(\mathrm{mK})( \pm 1)(\mathrm{Raw})$ & $($ Corr. $)$ & FWHM $(\mathrm{MHz})$ & $\mathrm{kms}^{-1}$ & Corr. \\
\hline 17992.56 & 1 & 71 & 887.01 & 905.1 & 1.322 & 22.04 & 28.9 \\
18045.89 & 2 & 89 & 219.21 & 230.7 & 1.382 & 23.53 & 32.0 \\
17569.58 & 6 & 128 & 26.15 & 29.4 & 1.536 & 26.15 & 38.1 \\
17645.81 & 8 & 140 & 16.19 & 16.4 & 1.243 & 21.18 & 26.8 \\
17696.06 & 10 & 150 & 6.80 & 9.4 & 1.754 & 29.87 & 47.2 \\
17613.65 & 12 & 159 & 4.62 & 6.3 & 1.736 & 29.56 & 46.6 \\
17606.87 & 13 & 163 & 3.47 & 4.6 & 1.708 & 3.09 \\
17702.32 & 15 & 170 & 2.54 & 2.2 & 1.795 & 45.2 \\
17588.21 & 17 & 177 & 2.22 & - & - & 21.10 & 26.9 \\
17612.76 & 18 & 180 & confused & & & - \\
17601.43 & 19 & 183 & $<1.0$ & & & \\
\hline
\end{tabular}

${ }^{1}$ assumes $\Delta \mathrm{V}($ Doppler $)=28.9 \mathrm{~km} \mathrm{~s}^{-1}$

${ }^{2}$ offset width $=32 \mathrm{~km} \mathrm{~s}^{-1}$

${ }^{3}$ confused with $\Delta n=12$ line 
TABLE 4

Lines Detected in Orion at 6 GHz. ${ }^{1,2,3,4,5}$

\begin{tabular}{|c|c|c|c|c|c|c|c|c|}
\hline Rest Freq $(\mathrm{MHz})$ & Obs Freq $(\mathrm{MHz})$ & ID & $\mathrm{T}_{A}(\mathrm{~K})$ & $\mathrm{T}_{A}$ (Corr.) & FWHM(MHz) & $\mathrm{kms}^{-1}$ (raw) & $\mathrm{kms}^{-1}$ (Corr.) & Note \\
\hline 5979.13 & $5979.25 \pm 0.03$ & $\mathrm{H} 194(\Delta n=7)$ & 0.0205 & 0.0205 & 0.368 & 18.4 & 21.6 & - \\
\hline 5981.33 & 5980.94 & $\mathrm{NO} ?$ & 0.0087 & 0.0087 & 0.336 & 16.8 & 19.3 & 6 \\
\hline 5982.45 & $5982.50 \pm 0.041$ & $\mathrm{H} 174(\Delta n=5)$ & 0.0547 & 0.0781 & 0.596 & 29.7 & 47.1 & - \\
\hline 5983.30 & - & $\mathrm{H} 286(\Delta n=24)$ & - & - & - & - & - & 7 \\
\hline 5984.89 & 5984.92 & $\operatorname{He} 174(\Delta n=5)$ & 0.003 & - & - & - & - & 8 \\
\hline 5985.70 & - & $\mathrm{H} 257(\Delta n=17)$ & - & - & - & - & - & 9 \\
\hline 5987.15 & $5987.18 \pm .003$ & $\mathrm{H} 129(\Delta n=2)$ & 0.797 & 0.822 & 0.443 & 22.1 & 29.5 & - \\
\hline - & 5988.59 & U5988.5 & 0.033 & 0.033 & 0.329 & 16.4 & 18.6 & 10 \\
\hline 5989.50 & - & $\mathrm{H} 266(\Delta n=19)$ & - & - & - & - & - & 11 \\
\hline 5989.59 & 5989.67 & $\mathrm{He} 129(\Delta n=2)$ & 0.0821 & 0.106 & 0.562 & 28.0 & 43.7 & \\
\hline 5991.18 & $5990.54 ?$ & $\mathrm{H} 224(\Delta n=11)$ & $\sim 0.004$ & - & - & - & - & 12 \\
\hline 5993.35 & 5992.99 & NO? & 0.0084 & 0.0084 & 0.373 & 18.6 & 22.1 & 13 \\
\hline 5994.48 & $5993.89 \pm .060$ & $\mathrm{H} 247(\Delta n=15)$ & 0.0039 & - & - & - & - & 14 \\
\hline - & 5994.59 & U5994.6 & 0.0120 & 0.0120 & 0.375 & 18.7 & 22.2 & 15 \\
\hline 5997.79 & $5997.43 \pm .05$ & $\mathrm{H} 252(\Delta n=16)$ & 0.0066 & 0.0068 & 0.456 & 22.7 & 30.7 & \\
\hline 6000.77 & - & $\mathrm{H} 282(\Delta n=23)$ & $<0.002$ & - & - & - & - & 16 \\
\hline 6002.22 & $6002.26 \pm .031$ & $\mathrm{H} 210(\Delta n=9)$ & 0.0124 & 0.0127 & 0.425 & 21.2 & 27.5 & \\
\hline 6003.45 & $6003.35 \pm 0.038$ & $\mathrm{H} 236(\Delta n=13)$ & 0.0066 & 0.0068 & 0.355 & 17.7 & 20.8 & \\
\hline 6005.63 & $\sim 6005$ & ${ }^{17} \mathrm{OH}^{2} \Pi_{3 / 2}$ & - & - & - & - & - & 17 \\
\hline 6006.66 & 6006.80 & $\mathrm{H} 270(\Delta n=20)$ & - & - & - & - & - & 18 \\
\hline - & 6009.03 & U6009.0 & 0.0078 & 0.0098 & 0.693 & 28.0 & 43.1 & 19 \\
\hline 6011.14 & - & $\mathrm{H} 278(\Delta n=22)$ & - & - & - & - & - & 20 \\
\hline 6011.40 & $6011.36 \pm .050$ & $\mathrm{H} 230(\Delta n=12)$ & 0.005 & 0.005 & 0.43 & 19.0 & 22.9 & 21 \\
\hline 6013.47 & $6013.04 \pm .11$ & $\mathrm{H} 274(\Delta n=21)$ & 0.0016 & 0.0016 & 0.464 & 21.5 & 28.2 & \\
\hline 6016.75 & 6016.24 & $\mathrm{OH}^{2} \Pi_{3 / 2}$ & 0.0018 & 0.0018 & 0.40 & 20.0 & 25.0 & 22 \\
\hline 6016.59 & $6016.91 \pm .11$ & $\mathrm{H} 217(\Delta n=10)$ & 0.0045 & 0.0048 & - & - & - & 23 \\
\hline 6019.23 & $6018.08 \pm .10$ & $\mathrm{H} 289(\Delta n=25)$ & 0.0006 & 0.0007 & 0.40 & 20.0 & 25.0 & 24 \\
\hline - & 6021.56 & U6021.5 & 0.0043 & 0.0046 & 0.485 & 34.1 & 56.8 & \\
\hline 6022.61 & $6022.74 \pm .038$ & $\mathrm{H} 202(\Delta n=8)$ & 0.0127 & 0.0163 & 0.561 & 28.0 & 43.4 & \\
\hline 6025.58 & $6025.62 \pm .003$ & $\mathrm{H} 147(\Delta n=3)$ & 0.2903 & 0.302 & 0.452 & 22.6 & 30.5 & \\
\hline 6027.20 & 6027.06 & $\mathrm{H} 261(\Delta n=18)$ & 0.0103 & 0.0104 & 0.394 & 19.6 & 24.2 & 25 \\
\hline 6028.04 & 6028.11 & $\operatorname{He} 147(\Delta n=3)$ & 0.0302 & 0.050 & 0.622 & 31.0 & 50.8 & \\
\hline 6030.75 & 6030.29 & $\mathrm{OH}^{2} \Pi_{3 / 2}$ & 0.0176 & 0.0176 & 0.203 & 10.1 & 10.1 & 26 \\
\hline 6035.09 & 6034.65 & $\mathrm{OH}^{2} \Pi_{3 / 2}$ & 0.0434 & 0.0434 & 0.211 & 10.5 & 10.5 & 27 \\
\hline 6035.27 & - & $\mathrm{OH} ?$ & 0.0103 & 0.0103 & 0.104 & 5.81 & 5.8 & \\
\hline 6036.96 & $6037.04 \pm .02$ & $\mathrm{H} 184(\Delta n=6)$ & 0.0339 & 0.0413 & 0.546 & 27.2 & 41.2 & \\
\hline 6039.43 & 6039.61 & $\mathrm{He} 184(\Delta n=6)$ & 0.0045 & 0.0082 & 0.652 & 32.5 & 55.1 & \\
\hline - & 6042.4 & $\mathrm{U} 6042.4$ & 0.0025 & 0.0025 & 0.142 & 7.1 & 7.2 & \\
\hline 6045.52 & $6045.50 \pm .070$ & $\mathrm{H} 241(\Delta n=14)$ & 0.003 & 0.003 & 0.39 & 19.5 & 24.2 & \\
\hline 6106.86 & $6106.89 \pm .0008$ & $\mathrm{H} 102(\Delta n=1)$ & 3.588 & 3.661 & 0.422 & 21.05 & 27.3 & \\
\hline 6109.35 & 6109.39 & $\operatorname{He} 102(\Delta n=1)$ & 0.408 & 0.429 & 0.469 & 23.4 & 32.0 & \\
\hline
\end{tabular}


TABLE 5

Notes to TABle 4.

\begin{tabular}{|c|c|}
\hline Note number & Note \\
\hline 1 & Offset width $=8$ channels $=0.624 \mathrm{MHz}=31.15 \mathrm{kms}^{-1}$ \\
\hline 2 & assumes $\Delta V($ Dopp $)=27.8 \mathrm{kms}^{-1}$ for $\mathrm{H}(102,1)$ \\
\hline 3 & FWHM assumed for $\mathrm{He}(102,1)$ is $27.8 \mathrm{kms}^{-1}$ \\
\hline 4 & $1 \sigma \mathrm{rms}$ noise $<0.001$ for $5979<\nu<6005 \mathrm{MHz}$ \\
\hline 5 & $1 \sigma \mathrm{rms}$ noise $<0.0005$ for $6005<\nu<6050 \mathrm{MHz}$ \\
\hline 6 & $\mathrm{NO}$ at $\mathrm{V}_{L S R}$ of $+19 \mathrm{kms}^{-1}$ \\
\hline 7 & in wing of $\mathrm{H}(174,5)$ line \\
\hline 8 & in wing of $\mathrm{H}(129,2)$ line \\
\hline 9 & in wing of $\mathrm{H}(129,2)$ line \\
\hline 10 & Deuterium? $\mathrm{H}_{2}$ ? \\
\hline 11 & confused with $\mathrm{He}(129,2)$ line \\
\hline 12 & in wing of $\mathrm{He}(129,2)$ line (interference?) \\
\hline 13 & $\mathrm{NO}$ at $\mathrm{LSR}$ vel of $+18 \mathrm{kms}^{-1}$ \\
\hline 14 & not cleaned (confused) \\
\hline 15 & too strong for $\mathrm{H}(247,15)$ line \\
\hline 16 & not cleaned \\
\hline 17 & likely interference \\
\hline 18 & not cleaned \\
\hline 19 & ${ }^{34} \mathrm{SO}_{2} ? \mathrm{CH}_{4} ?$ \\
\hline 20 & confused with $\mathrm{H}(230,12)$ \\
\hline 21 & no fit made, edge of window \\
\hline 22 & confused with $\mathrm{H}(217,10)$ \\
\hline 23 & confused with $\mathrm{OH}$ \\
\hline 24 & $1 \sigma$ feature \\
\hline 25 & Deuterium? ${ }^{3} \mathrm{He} ?$ \\
\hline 26 & at $\mathrm{V}_{L S R}$ of $23 \mathrm{kms}^{-1}$ \\
\hline 27 & at $\mathrm{V}_{L S R}$ of $22 \mathrm{kms}^{-1}$ \\
\hline
\end{tabular}

TABLE 6

Hydrogen Recombination Lines in W51 ${ }^{1}$ Between 5975 and $6050 \mathrm{MHz}$ with $\Delta n<20^{2}$.

\begin{tabular}{|c|c|c|c|c|c|c|c|}
\hline Frequency $(\mathrm{MHz})$ & $\Delta n$ & $n$ & $\mathrm{~T}_{A}(\mathrm{mK})( \pm 0.5)(\mathrm{Raw})$ & (Corr.) & FWHM(MHz) & $\mathrm{kms}^{-1}$ (raw) & $\mathrm{kms}^{-1}$ (Corr. \\
\hline 6106.856 & 1 & 102 & 1174.0 & 1235.4 & 0.450 & 22.10 & 31.5 \\
\hline 5987.147 & 2 & 129 & 235.72 & 250.8 & 0.452 & 22.64 & 32.2 \\
\hline 6025.584 & 3 & 147 & 95.15 & 101.2 & 0.454 & 22.60 & 32.1 \\
\hline 5982.446 & 5 & 174 & 25.64 & 29.1 & 0.495 & 24.82 & 37.5 \\
\hline 6036.964 & 6 & 184 & 14.24 & 16.2 & 0.495 & 24.59 & 37.0 \\
\hline 5979.132 & 7 & 194 & 9.70 & 11.5 & 0.517 & 25.93 & 40.1 \\
\hline 6022.606 & 8 & 202 & 7.15 & 8.6 & 0.523 & 26.04 & 40.6 \\
\hline 6002.216 & 9 & 210 & 5.77 & 6.6 & 0.495 & 24.73 & 37.4 \\
\hline 6016.590 & 10 & 217 & - & - & - & - & confused with $\mathrm{OH}$ \\
\hline 5991.178 & 11 & 224 & 3.75 & 4.4 & 0.506 & 25.33 & 38.4 \\
\hline 6011.398 & 12 & 230 & 2.93 & 3.1 & 0.436 & 21.75 & 30.6 \\
\hline 6003.451 & 13 & 236 & 2.14 & 2.1 & 0.305 & 15.24 & 17.7 \\
\hline 6045.523 & 14 & 241 & 3.19 & 3.2 & 0.334 & 16.57 & 20.1 \\
\hline 5994.475 & 15 & 247 & - & - & - & - & may contain interference \\
\hline 5997.790 & 16 & 252 & 1.23 & - & 0.546 & 27.31 & confused \\
\hline 5985.696 & 17 & 257 & - & - & - & - & in clean area of $\Delta n=2$ \\
\hline 6027.198 & 18 & 261 & - & - & - & - & in clean area of $\Delta n=3$ \\
\hline 5989.503 & 19 & 266 & - & - & - & - & confused with $\mathrm{He} \Delta n=2$ \\
\hline 6006.661 & 20 & 270 & - & - & - & - & close to interference \\
\hline
\end{tabular}

${ }^{1}$ assumes $\Delta \mathrm{V}($ Doppler $)=31.5 \mathrm{~km} \mathrm{~s}^{-1}$

${ }^{2}$ offset width $=31.15 \mathrm{~km} \mathrm{~s}^{-1}$ 
TABLE 7

Helium Recombination Lines in W51 Between 5975 And 6050 MHz with $\Delta n<9$.

\begin{tabular}{|c|c|c|c|c|c|c|}
\hline Frequency $(\mathrm{MHz})$ & $\Delta n$ & $n$ & $\mathrm{~T}_{A}(\mathrm{mK})$ & Rel. Line Area & FWHM(MHz) & comments \\
\hline 5981.573 & 7 & 194 & - & - & - & in clean area of $\mathrm{H}(174,5)$ \\
\hline 5984.888 & 5 & 174 & 1.69 & 0.28 & 0.156 & in clean area of $\mathrm{H}(129,2)$ \\
\hline 5989.591 & 2 & 129 & 17.06 & 8.45 & 0.465 & - \\
\hline 6025.065 & 8 & 202 & - & - & - & confused with $\mathrm{H}(147,3)$ line \\
\hline 6028.044 & 3 & 147 & 6.43 & 2.64 & 0.387 & - \\
\hline 6039.429 & 6 & 184 & $<1$ & - & - & - \\
\hline
\end{tabular}

TABLE 8

Other Lines Detected in W51 Near 6.0 GHz.

\begin{tabular}{lll}
\hline \hline Frequency $(\mathrm{MHz})$ & $\mathrm{T}_{A}(\mathrm{~K})$ & \multicolumn{1}{c}{ Identification } \\
\hline 5992.3 & -0.005 & possible interference \\
5994.5 & -0.004 & possible interference \\
5996.7 & 0.002 & U5996.7 \\
5999.0 & 0.003 & U5999.0 \\
6000.0 & 0.002 & $\mathrm{U} 6000.0$ \\
6016.7 & -0.007 & $\mathrm{OH}$ \\
6023.8 & 0.0036 & $\mathrm{U} 6023.8$ \\
6030.5 & -0.07 & $\mathrm{OH}$ \\
6030.7 & 0.034 & $\mathrm{OH}$ \\
6035.1 & 0.34 & $\mathrm{OH}$ \\
6042.4 & 0.003 & $\mathrm{U} 6042.4$ \\
\hline
\end{tabular}




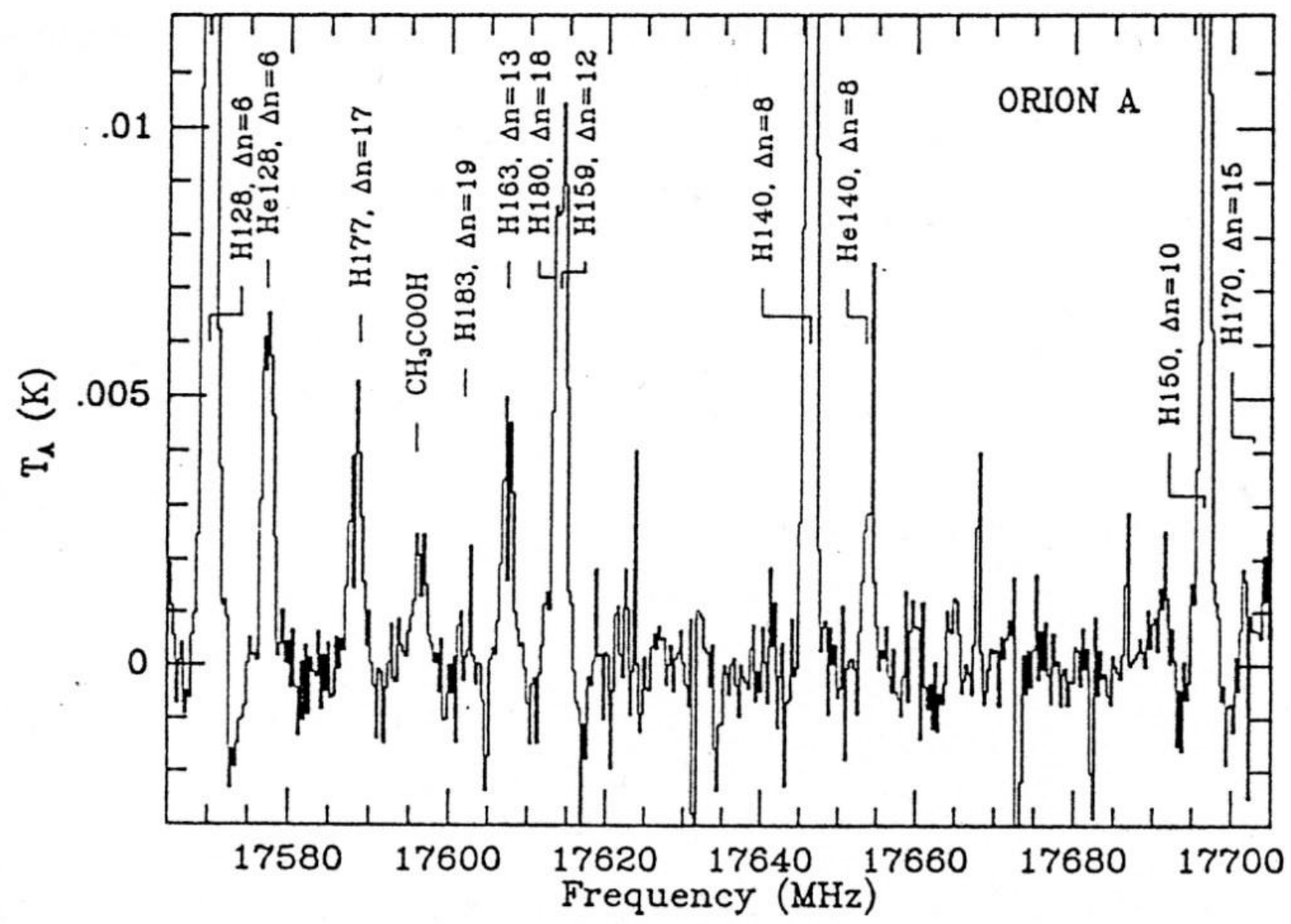

Fig. 1.- Spectrum of Orion A at 17.6 GHz obtained using frequency switching and presented after reference lines have been "cleaned" off. No sinusoids or polynomials have been removed from the baseline. Locations of the hydrogen and relevant helium lines are indicated. The $\mathrm{H}(163,13)$ line appears to be too weak compared to the $\Delta n=12$ and 17 lines and may be confused with a line, or lines, of molecular origin such as the $\mathrm{H}_{2}^{+}$ doublet at 17604.3 and $17610.3 \mathrm{MHz}$ (see text). Although an LSR velocity of $8 \mathrm{kms}^{-1}$ was assumed when the data were obtained, the detected frequencies in Table 2 have been adjusted to an LSR velocity of 0 $\mathrm{kms}^{-1}$ to agree with the value assumed for the $6 \mathrm{GHz}$ Orion data. 


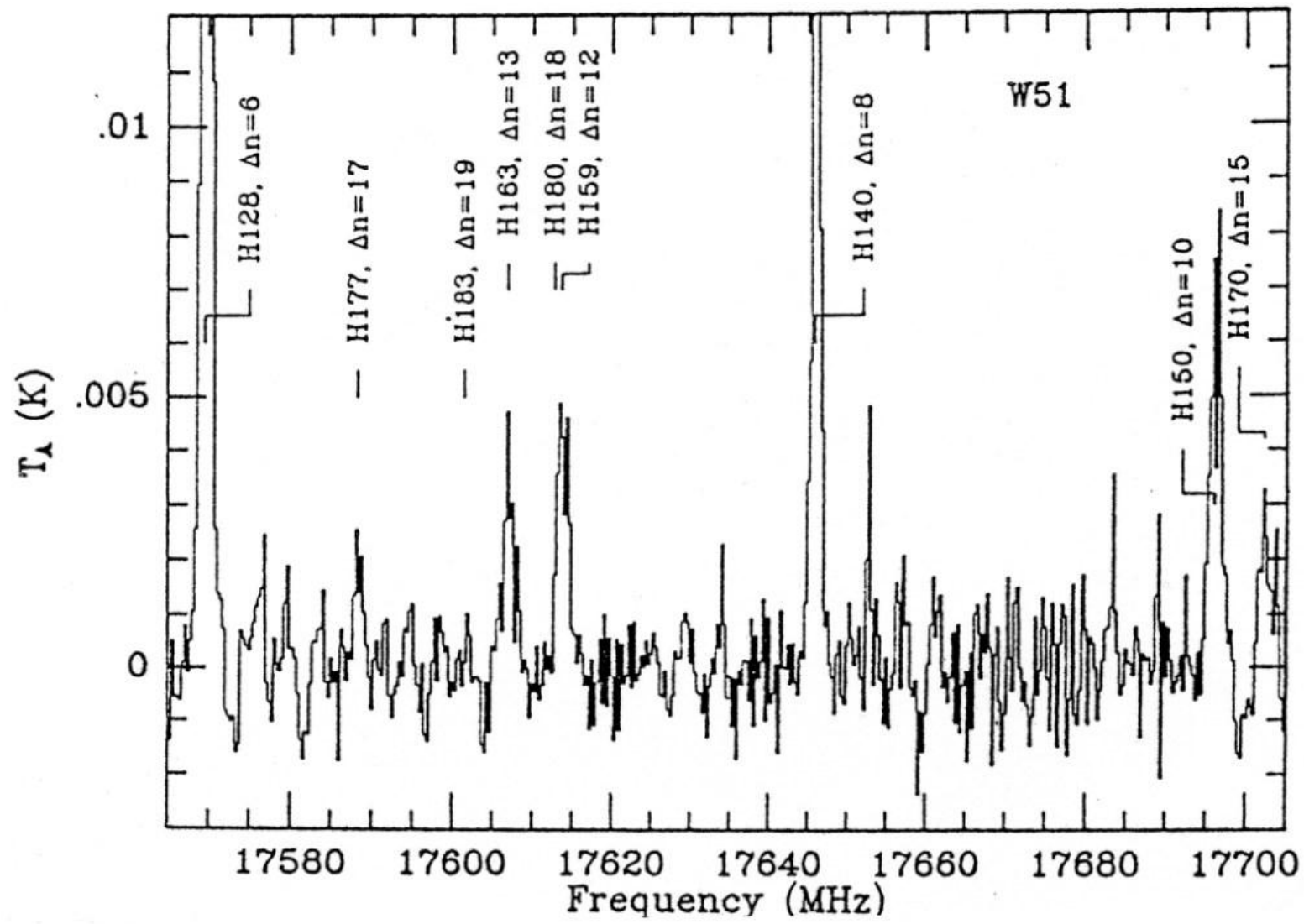

Fig. 2.- Spectrum of W51 at $17.6 \mathrm{GHz}$, after frequency-switched reference lines have been removed. The velocity assumed was $\mathrm{V}_{L S R}=60 \mathrm{kms}^{-1}$. The slight blueshift in the line position indicates that the true velocity is approximately $\mathrm{V}_{L S R}=58 \mathrm{kms}^{-1}$. No sinusoids or polynomials have been removed from the baseline. 


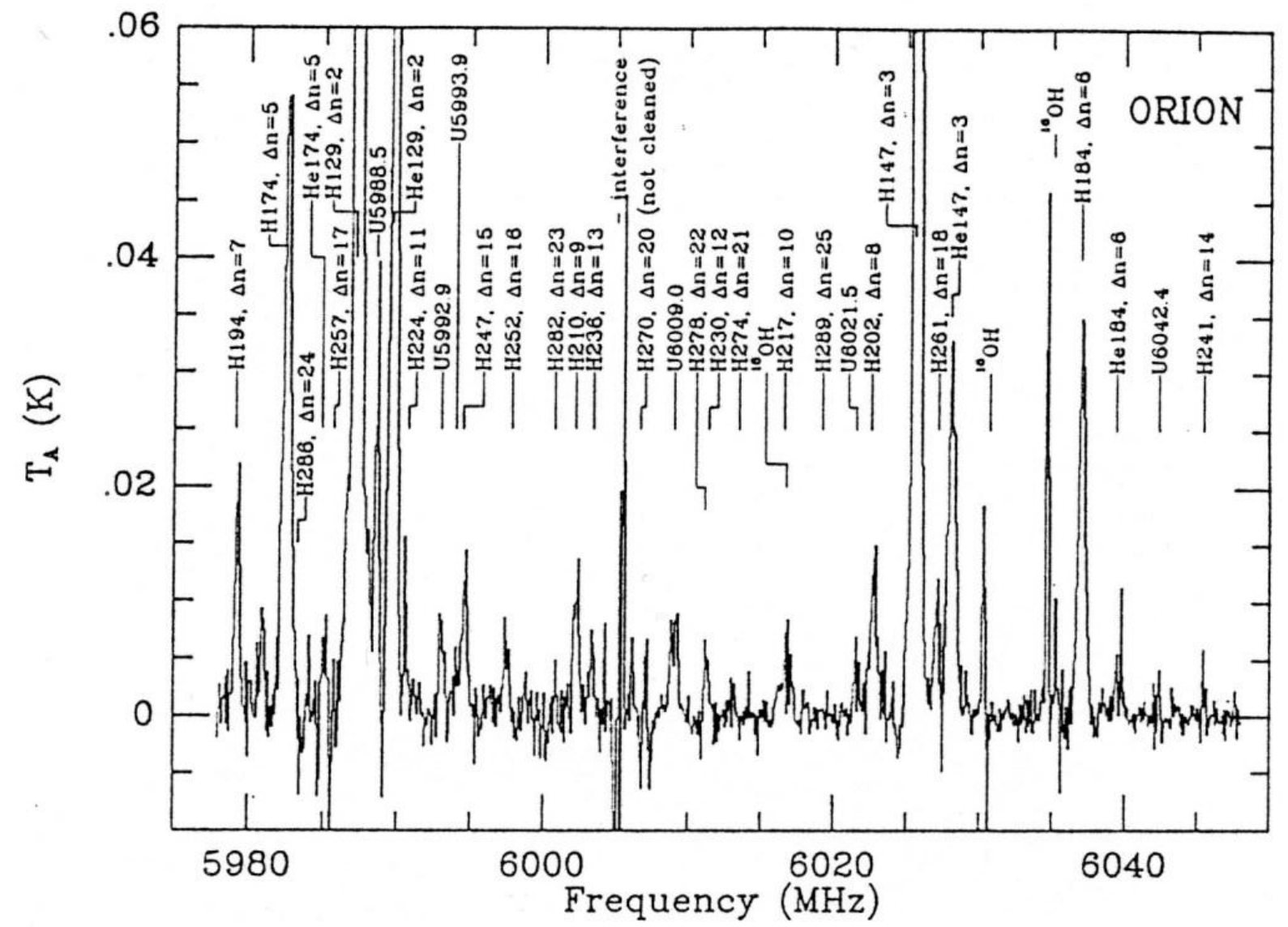

Fig. 3.- Spectrum of Orion at $6 \mathrm{GHz}$. No sinusoids or polynomials have been removed. The expected location of recombination lines with $20<\Delta n<30$ have been indicated for information purposes only and this is not meant to imply that they have been detected. An LSR velocity of $0 \mathrm{kms}^{-1}$ was assumed. 


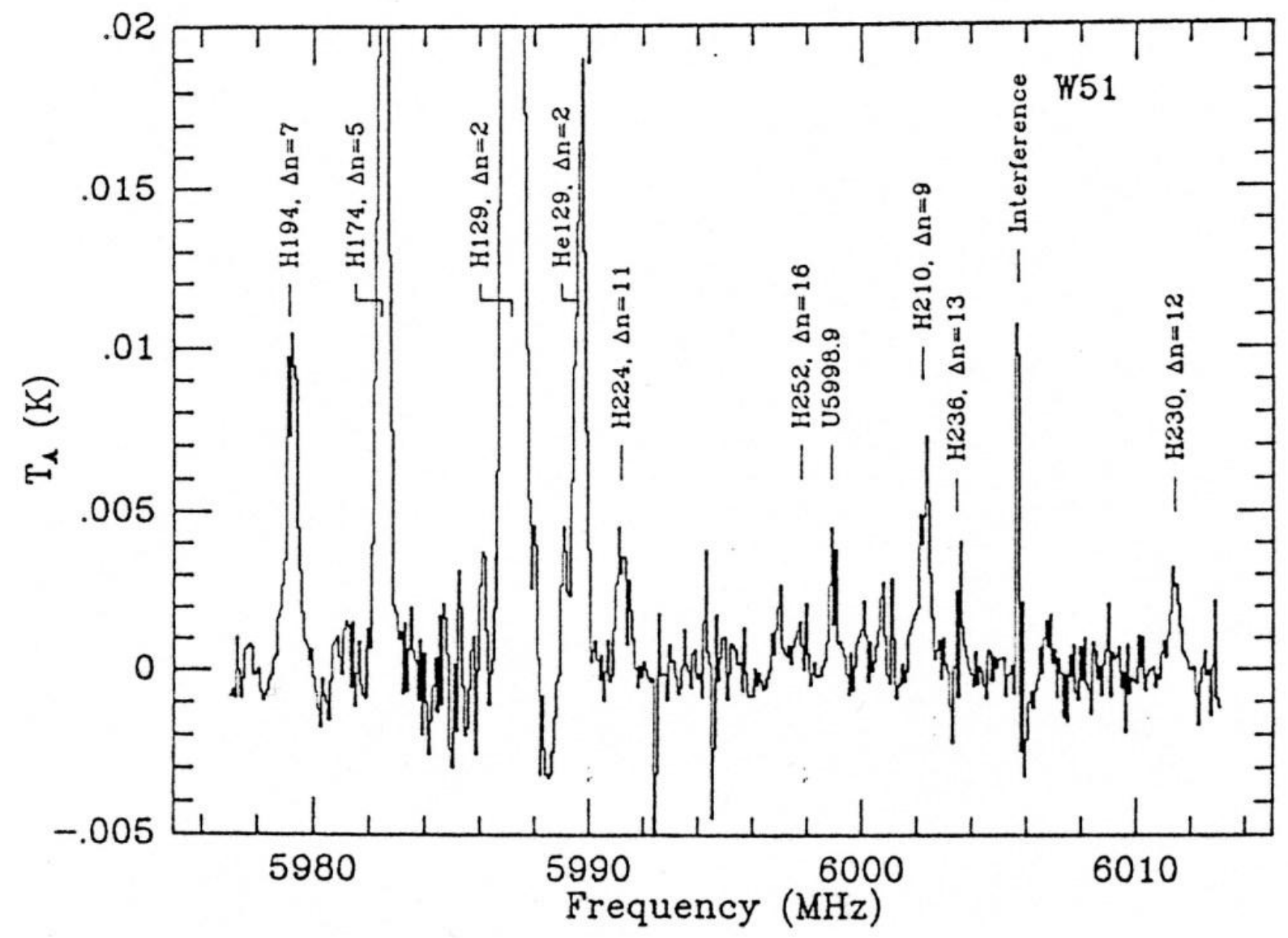

Fig. 4. - Spectrum of W51 below $6010 \mathrm{GHz}$ showing location of hydrogen and helium recombination lines as well as several molecular and U-lines. The frequency-switched reference lines have been removed but no sinusoids or polynomials have been removed from the baseline. 


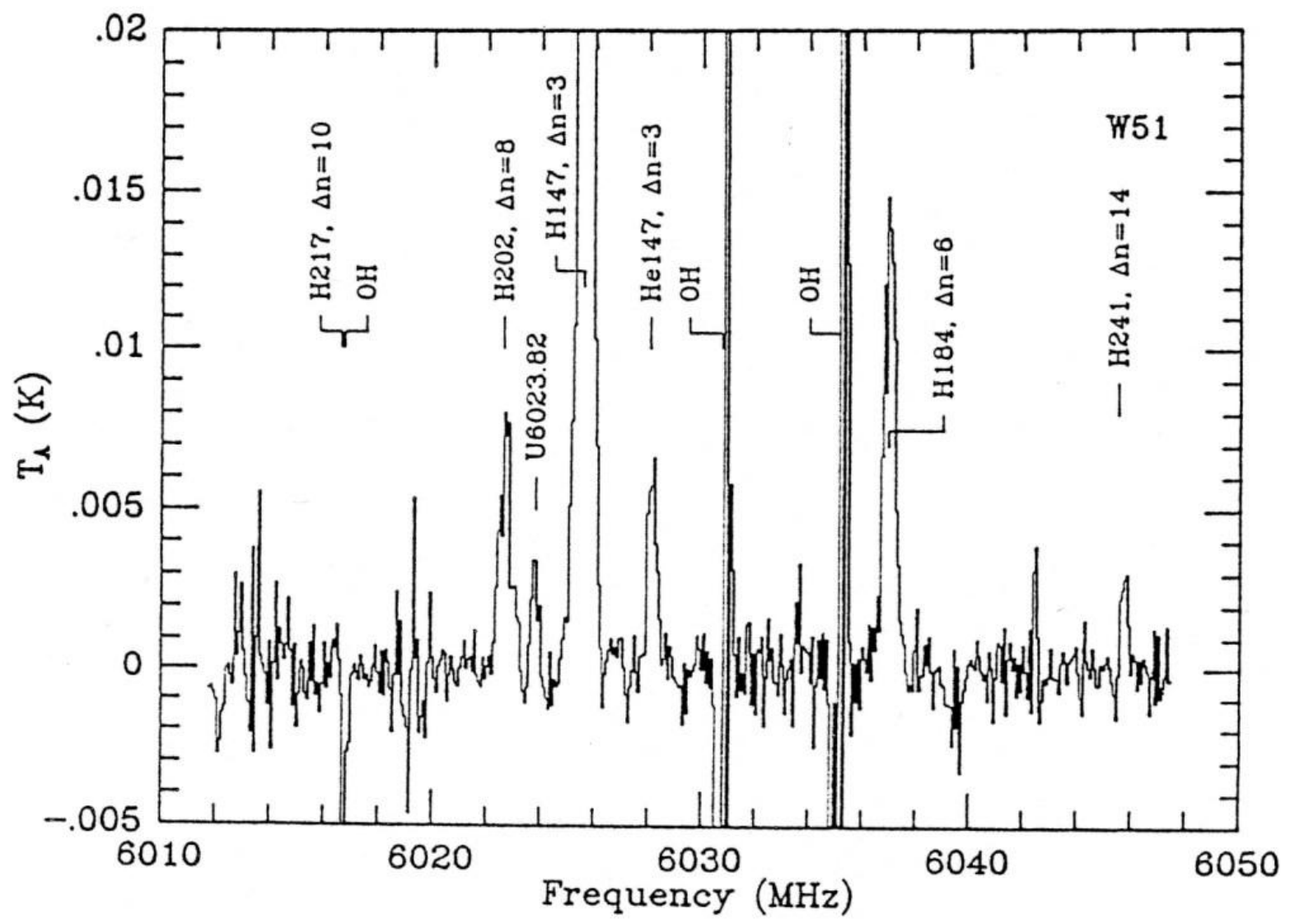

Fig. 5.- Spectrum of W51 above $6010 \mathrm{MHz}$. The frequency-switched reference lines have been removed but no sinusoids or polynomials have been removed from the baseline. 


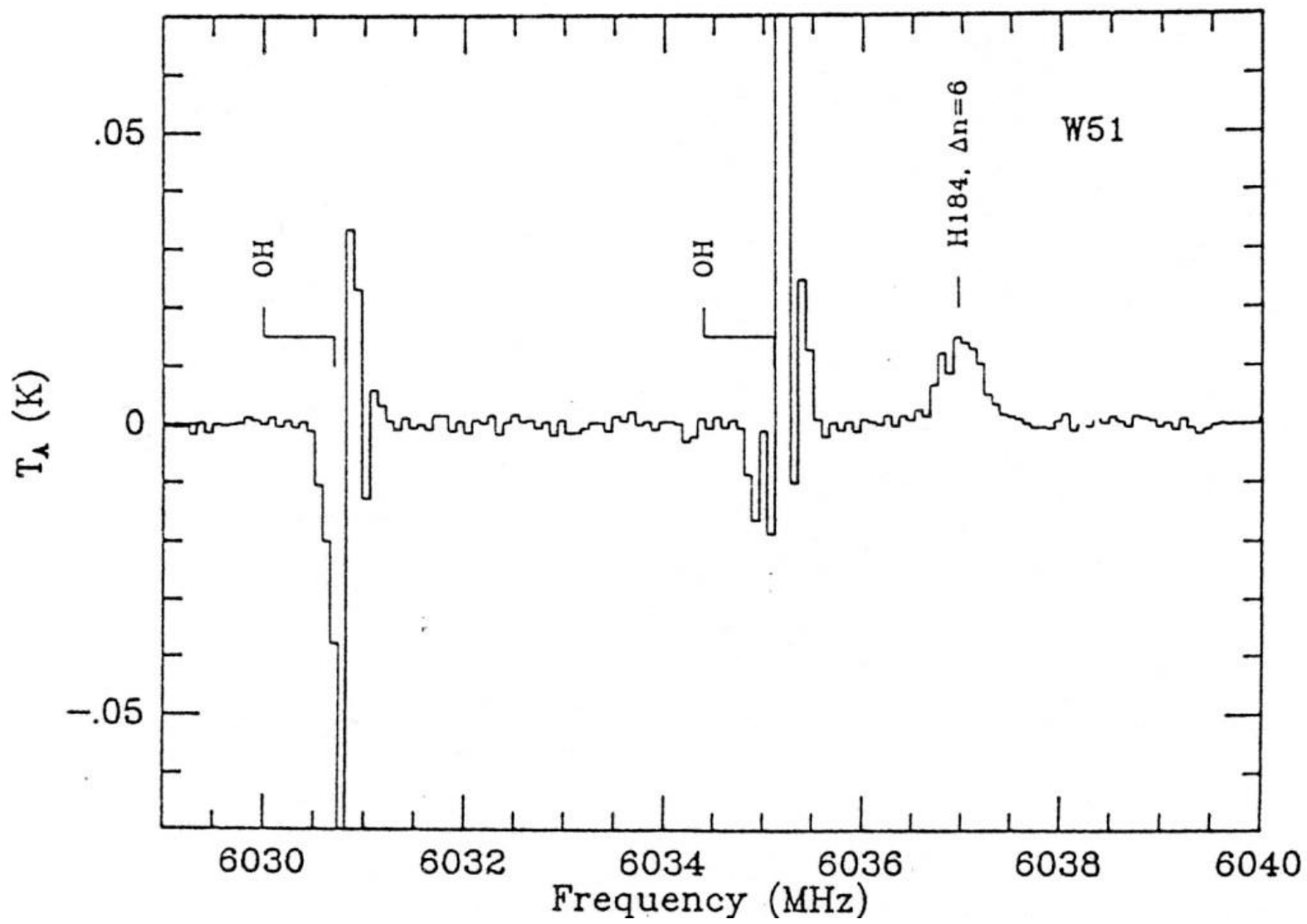

Fig. 6.- A portion of the spectrum of W51 at $6 \mathrm{GHz}$ showing the two strongest $\mathrm{OH}$ lines and the $\mathrm{H}(184,6)$ line. Structure is visible in the red wing of all three lines. 

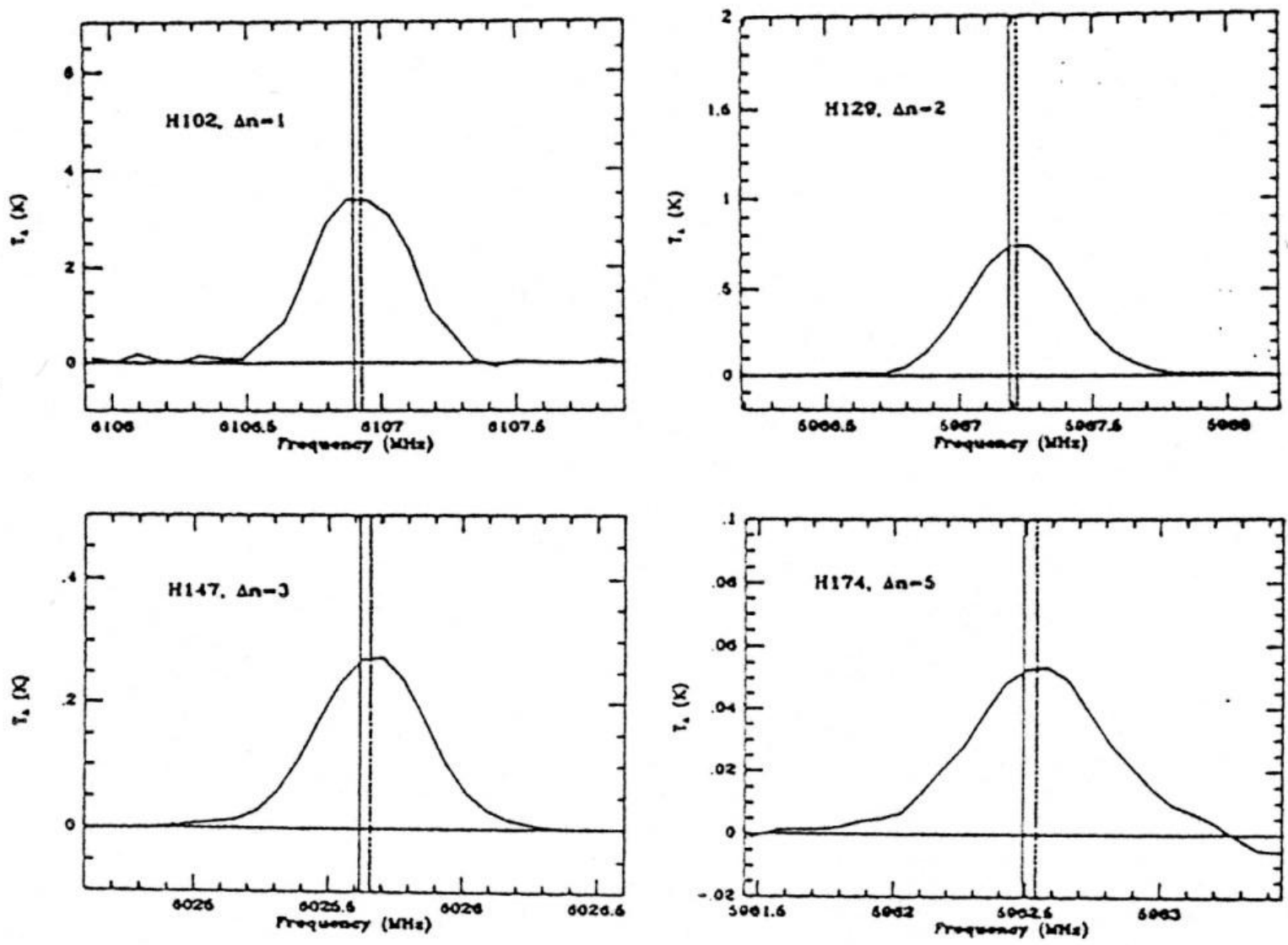

Fig. 7.- Plots of individual recombination lines observed in Orion at $6 \mathrm{GHz}$ showing the relative position of the line center frequencies. The solid line indicates the predicted position of the line. The dashed line indicates the observed line center. 

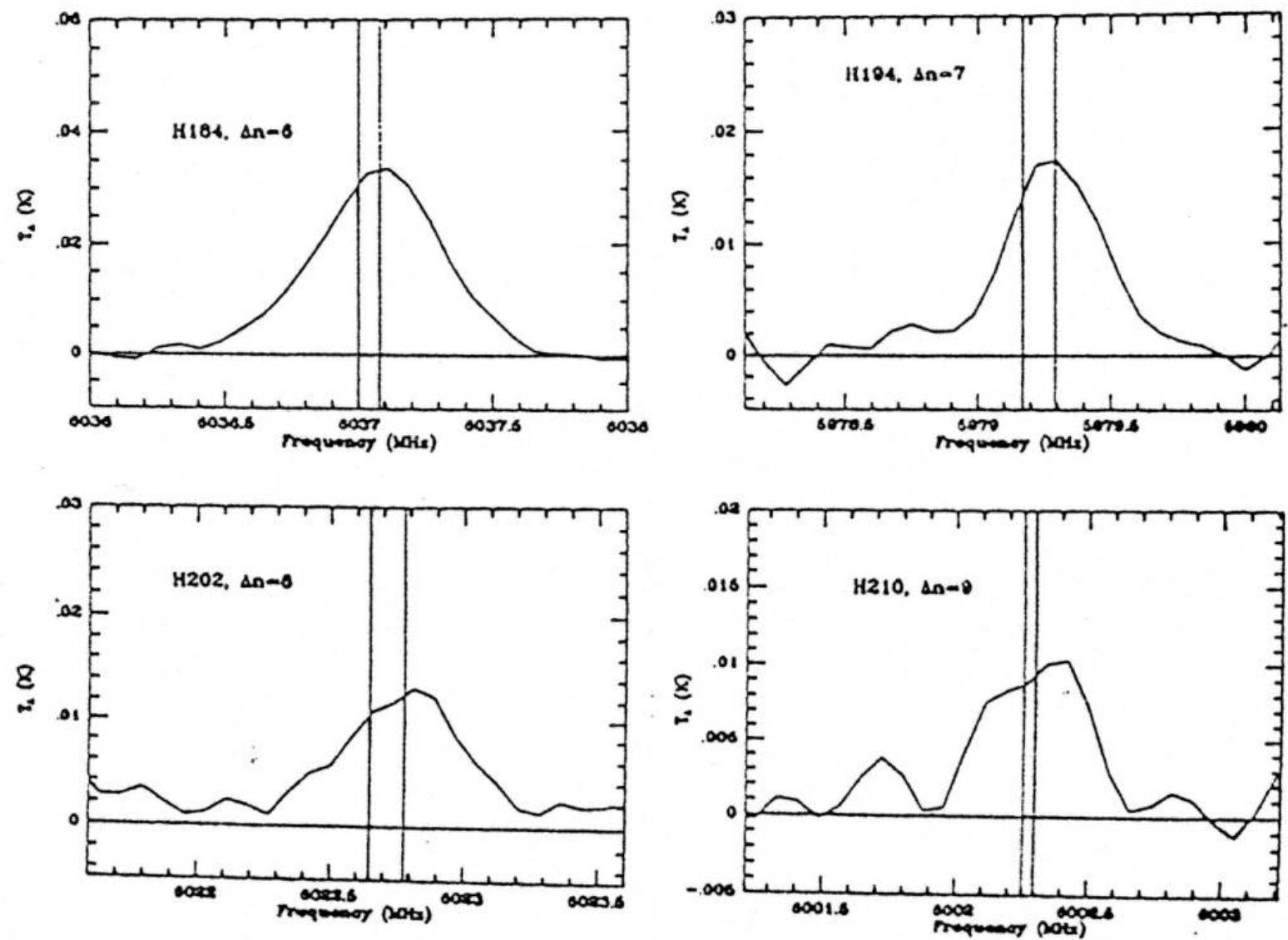

Fig. 8.- Same as Fig 7. 

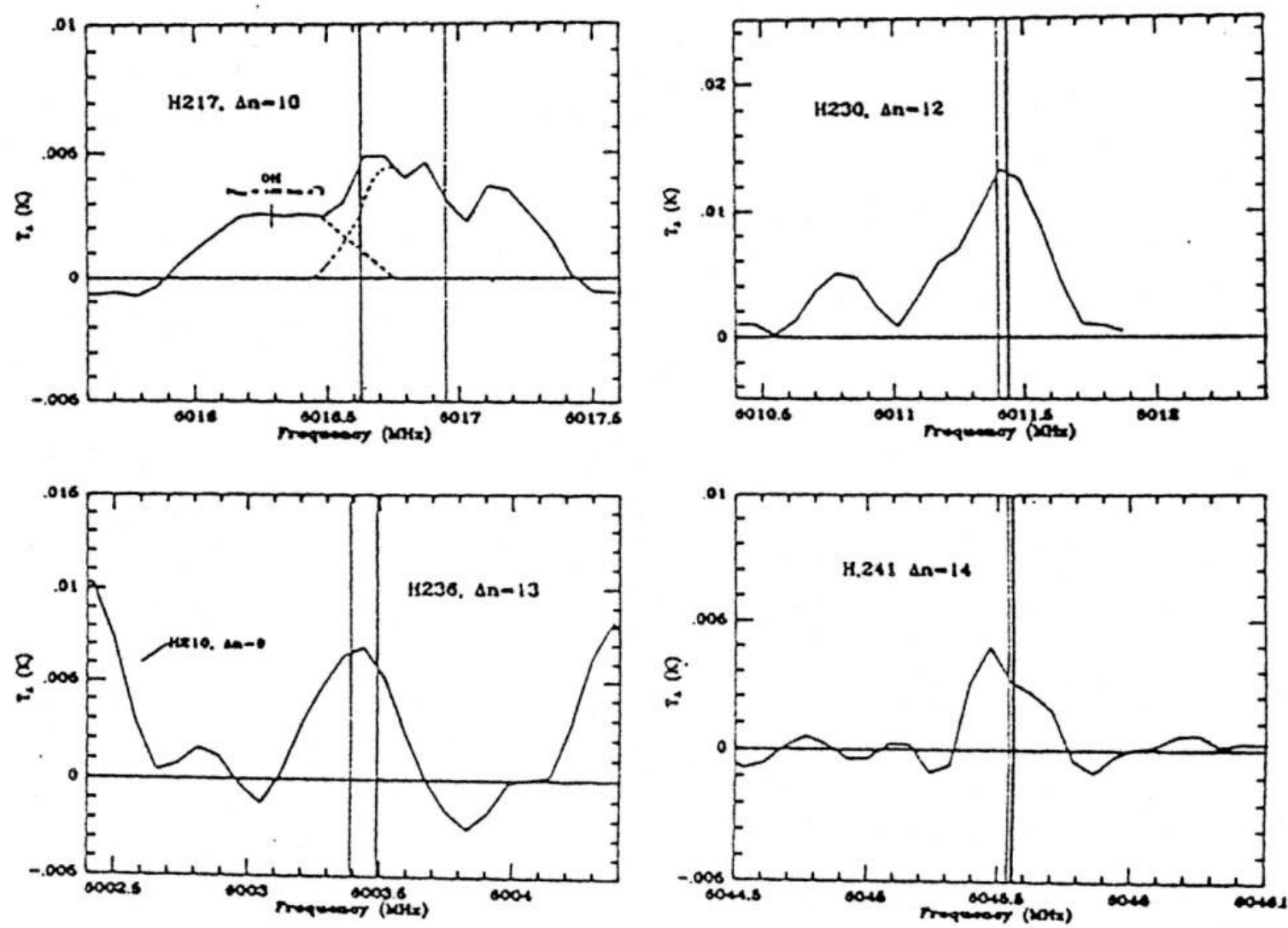

Fig. 9.- Same as Fig 7. Because the $\Delta n=10$ line is confused with an $\mathrm{OH}$ line its amplitude and width are uncertain and dashed curves have been drawn to indicate the assumptions made in determining the line position. 

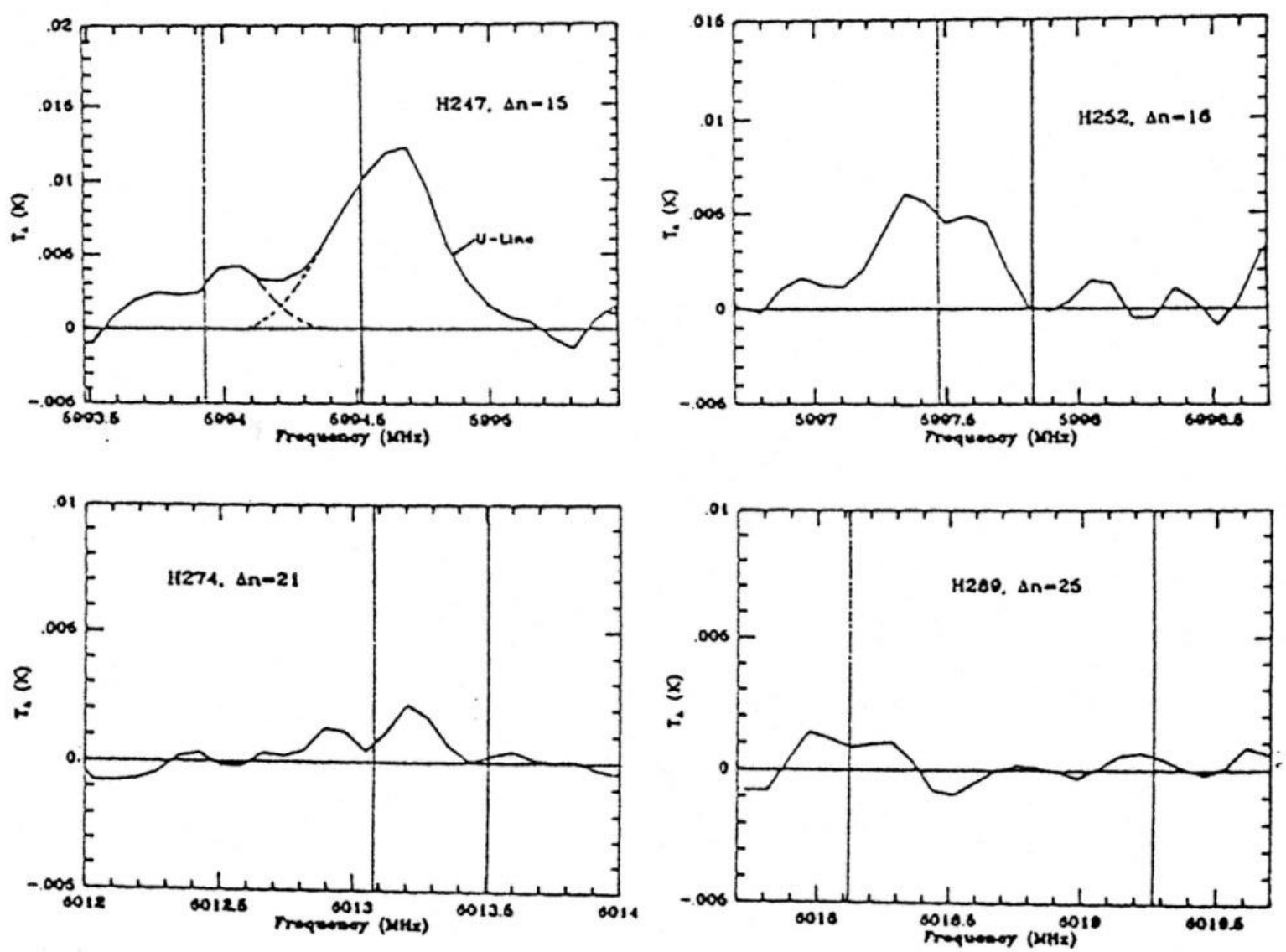

Fig. 10.- Same as Fig 7. Although the $\Delta n=25$ line has been included its identification is questionable. 


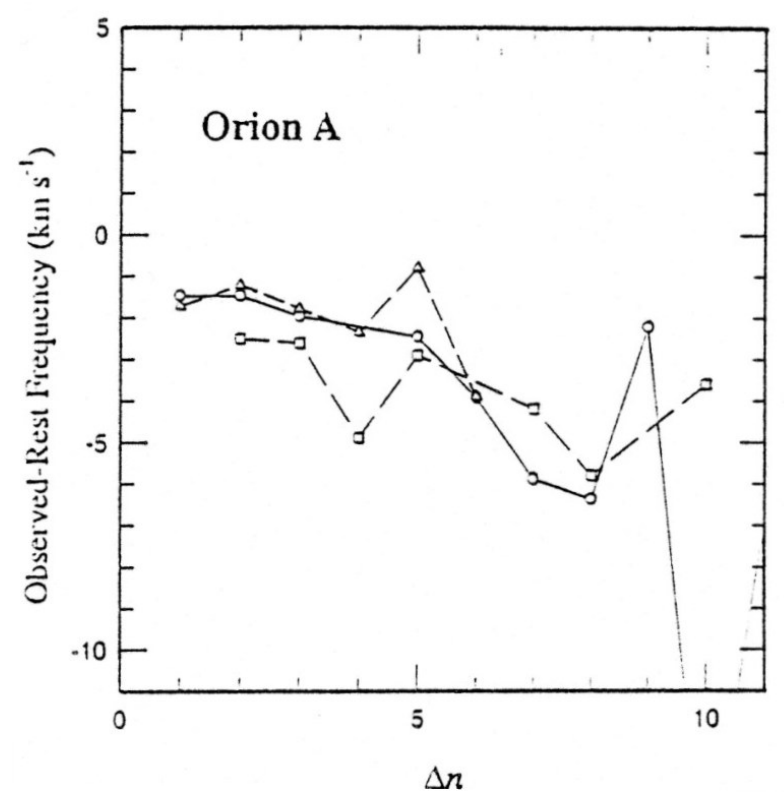

Fig. 11. - The differences between observed and rest frequencies (expressed in velocity units) for lines near $6 \mathrm{GHz}$ in Orion with $\Delta n$-values $<11$. (circles) data from this paper, (square) data from Rood et al (1984), (triangle) data from Smirnov et al (1984).

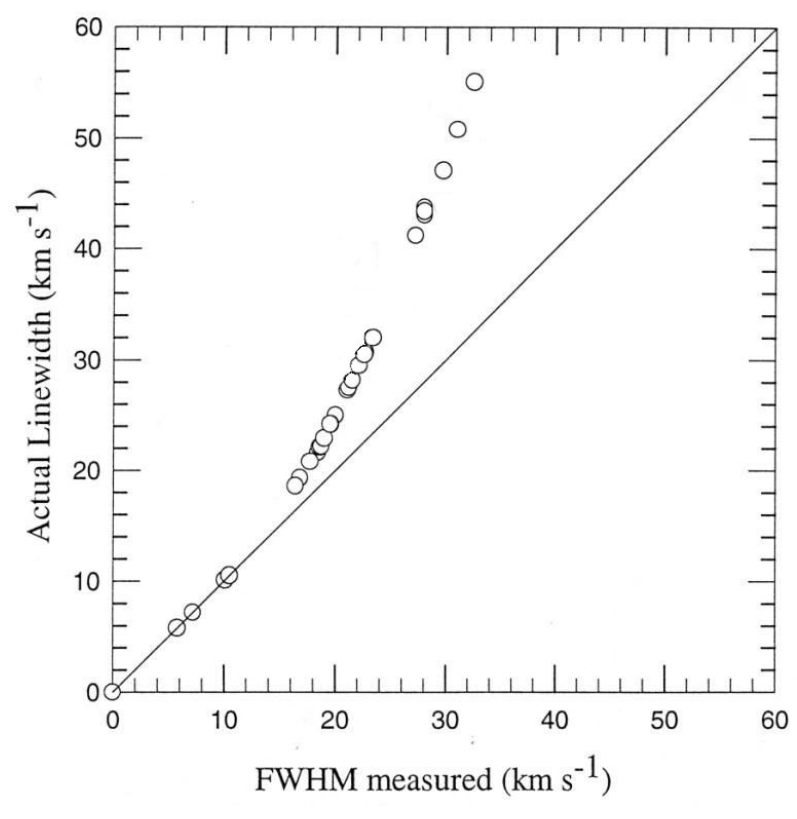

Fig. 12.- The curve defined by the open circles is used to obtain the true width of a line from the measured linewidth when frequency-switched offsets of $\pm 15.5 \mathrm{kms}^{-1}$ are used. (see Appendix A).

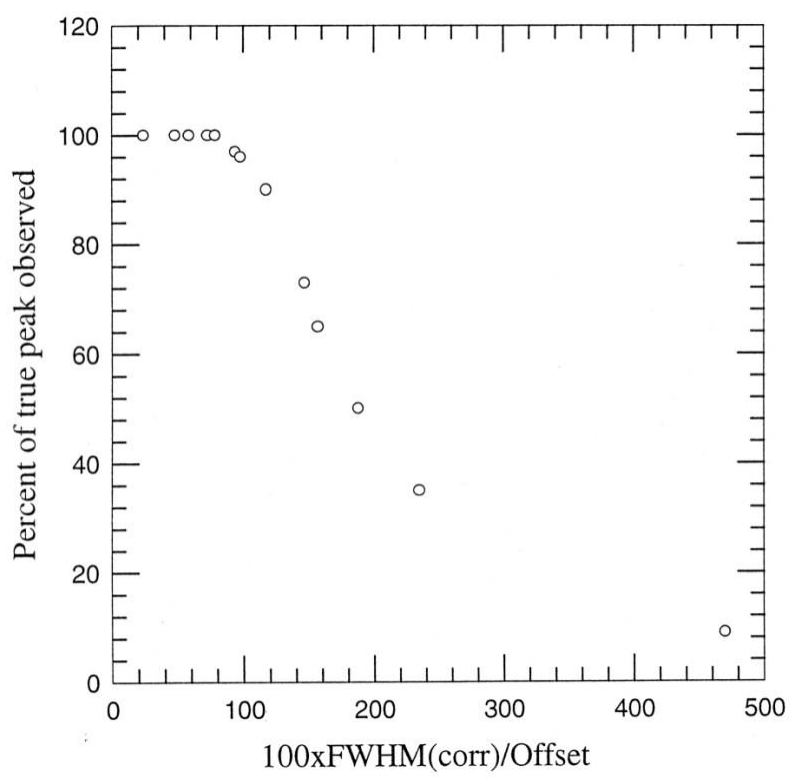

Fig. 13. - Curve used to obtain the true peak line strength of a wide, frequency-switched line when the true width of the line is known. 


\section{APPENDIX A: CORRECTION CURVES TO RECOVER THE TRUE LINE PARAMETERS OF WIDE, FREQUENCY-SWITCHED LINES.}

It is important to have flat baselines for the LINECLEAN program to work effectively and, to achieve this, it was necessary to place the frequency-switched reference line close to the signal line. The width of impact-broadened lines can then be a significant fraction of the frequency-switched interval. When this happens, the measured linewidths, and possibly even the peak values, can be reduced because one wing of the reference line falls on top of the signal line. Correction curves are therefore required so the true line strengths and widths can be recovered.

To obtain the information needed to correct for this effect, $\mathrm{H} \alpha$ lines in Orion A and W51 were observed with increasingly narrower frequency-switched offsets to simulate the effect produced by linewidths that become increasingly more broadened by impact broadening and fill more of the frequency-switched window. The correction curve appropriate for a frequency-switched offset of $\sim 31 \mathrm{~km} \mathrm{~s}^{-1}$ using the MOR data reduction technique described by Bell (1997) with 5 overlaps, is given by the open circles in Fig. 12. The method used to obtain this detailed curve is complicated, and will not be discussed here, since it turns out that a perfectly adequate curve can be obtained simply by comparing the measured frequency-switched widths to re-observed, or previously-published widths, obtained on a few sources with different intrinsic linewidths (using position switching or very wide frequency-switched offsets). Although the uncertainty in the curve was expected to increase slightly as the linewidth increased, it was shown by Bell et al. (2000) that the corrected linewidths obtained using the curve in Fig 12 agree well with results obtained by others using position switching, even for the widest lines. Furthermore, there appears to be no reason why the curve in Fig. 12 cannot be easily and accurately scaled to fit any frequency-switched offset by comparing the measured linewidths obtained using the new offset to a few known linewidths.

Once the corrected line widths have been determined, the corrected peak values can be obtained directly from the curve in Fig. 13. This curve is easily obtained by observing any strong line of known width with several different frequency-switched offsets.

Although the correction curves presented here have been included for illustration purposes, observers wishing to use this technique may wish to derive their own. 